\title{
Analysis and Simulation of Distributed Nonlinearities in Ferroelectrics and Superconductors for Microwave Applications
}

\author{
David Seron, Carlos Collado, Member, IEEE, Jordi Mateu, Member, IEEE, and \\ Juan M. O'Callaghan, Senior Member, IEEE
}

\begin{abstract}
This paper gives closed-form equations for the intermodulation and third harmonic signals generated in a nonlinear transmission line with distributed quadratic nonlinearities in the conductor and dielectric. Although the formulation developed is general, it is intended to be used in planar devices combining hightemperature superconductor (HTS) and oxide ferroelectrics. The analysis in this paper shows that the intermodulation and third harmonic signals produced by an HTS tend to cancel those of a ferroelectric, and that full cancellation is theoretically possible. This opens the way for using HTS/ferroelectric multilayers, not (only) for their tunable or phase-shifting properties, but for highly linear spurious-free planar HTS devices.
\end{abstract}

Index Terms-Ferroelectric, high-temperature superconductor (HTS), intermodulation distortion (IMD), nonlinear transmission line (NLTL), nonlinearities, superconductor.

\section{INTRODUCTION}

$\mathbf{N}$ OVEL OXIDE materials such as superconductors and ferroelectrics have already shown their potential in high-performance microwave devices such as filters with low loss and high selectivity using oxide superconductors, or tunable filters and variable phase shifters made with ferroelectric materials in the paraelectric phase [1]. The combination of high-temperature superconductors (HTSs) and oxide ferroelectrics is also being considered to produce selective tunable filters with low loss [2], [3]. However, these expectations are somewhat limited by nonlinearities arising from the use of these materials, either when used alone (such as in an HTS filter) [4], [5] or in combination (such as in an HTS tunable filter using oxide ferroelectrics). Being able to predict and minimize the nonlinear response is thus of great importance to design devices that could take full advantage of the unique properties of these materials.

In this paper, we propose a method to analyze and predict nonlinearities taking place in devices having superconductors

Manuscript received August 9, 2005; revised October 20, 2005. This work was supported by the Spanish Ministry of Science and Technology under Project MAT2005-05656-C04-03.

D. Seron, C. Collado, and J. M. O'Callaghan are with the Departamento de Teoría de la Señal y Comunicaciones, Universitat Politécnica de Catalunya, Barcelona 08034, Spain (e-mail: collado@tsc.upc.edu; joano@tsc.upc.edu).

J. Mateu was with the Departamento de Teoría de la Señal y Comunicaciones, Universitat Politécnica de Catalunya, Barcelona 08034, Spain and also with the Centre Tecnològic de Telecommunicacions de Catalunya, Barcelona 08034, Spain. He is now with the National Institute of Standards and Technology, Boulder, CO 80305 USA.

Digital Object Identifier 10.1109/TMTT.2005.864110 and ferroelectric materials. We find closed-form equations for the intermodulation distortion (IMD) and third harmonic generation ( $3 \mathrm{rdH})$ occurring in traveling-wave and resonant transmission lines (TLs). These equations are validated with harmonic-balance (HB) simulations.

The analysis is useful to make quantitative assessments of the combined effects of HTS and ferroelectrics in IMD and $3 \mathrm{rdH}$. In particular, the analysis allows finding the conditions under which the nonlinear effects of the HTS can be compensated with those of the ferroelectric. If these conditions could be translated to an experimental device (which, for the case of a planar circuit including an HTS/ferroelectric bilayer would involve aspects such as ferroelectric thickness and proper dc electric-field bias), the implications for system performance would be important since it would be a way to obtain highly selective and highly linear HTS planar filters.

Even though this paper is centered in traveling-wave and resonant TLs, the extension of these results to more sophisticated devices such as filters and other resonators may be done following procedures similar to those described in [6].

\section{DiELECTRIC AND SUPERCONDUCTING NONLINEARITIES IN TLS}

In this paper, we will analyze the IMD and 3rdH occurring in a nonlinear transmission line (NLTL), either in a traveling wave or in a resonant configuration. We assume that the sources of nonlinearity are within the conductor and in the dielectric, as would be the case in an HTS/ferroelectric bilayer film grown on a dielectric substrate such as $\mathrm{LaAlO}_{3}$ [3]. The analysis is also applicable when only the HTS produces nonlinear effects (as it happens when using an HTS on a standard dielectric crystal). Likewise, the analysis can also be used when the nonlinearities are only due to the ferroelectric such as in planar circuits made with $\mathrm{Ba}_{x} \mathrm{Sr}_{1-x} \mathrm{TiO}_{3}$ (BSTO) on $\mathrm{LaAlO}_{3}$ and a metal conductor layer [3], [7].

The analysis below equally applies to incipient ferroelectrics [8] like STO, displacement-type ferroelectrics [9] like BSTO, or even the effects of the $\mathrm{MgO}$ substrate used in planar HTS circuits where the $\mathrm{MgO}$ may behave nonlinearly in certain conditions [10].

In Sections II-A and B, we detail the origin of nonlinearities in HTS and ferroelectric materials, and we discuss their impact on an NLTL. 


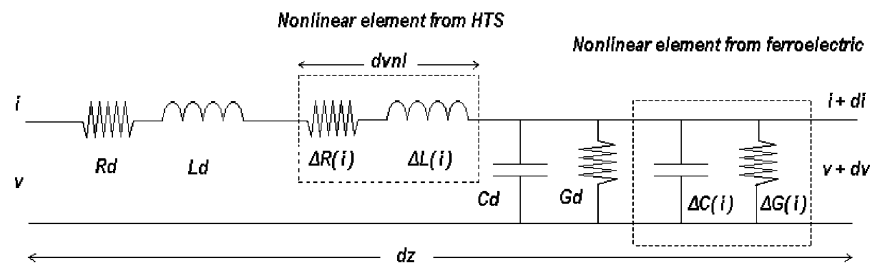

Fig. 1. Telegrapher's model of an elemental segment of nonlinear TL of length $d z$ made of HTS and ferroelectric materials.

\section{A. HTS Nonlinearities: Origins and Modeling}

The basic source of microwave nonlinearities in superconductors is the nonlinear dependence of the penetration depth and the quasi-particle conductivity on the microwave current (or microwave magnetic field) [11], [12]. While this intrinsic contribution sets the minimum level of nonlinearities [13], other extrinsic nonlinearities due to material defects may further enhance nonlinear distortions [14]-[16].

IMD and $3 \mathrm{rdH}$ are sensitive probes of the nonlinear effects, even at very low power for which other direct power-dependent measurements would observe a linear response [17], [18]. In most cases, HTS have an IMD power response that varies as the third order of the input microwave power, but other dependencies are also frequently observed [18]. In this paper, we will just consider this dependency, although other variations could be considered as well following the steps described in [6].

The nonlinear distortions produced by an HTS material used as a conductor in a (quasi) TEM TL (either traveling wave or resonant) may be modeled by a nonlinear resistance $(\Delta R(i))$ and inductance $(\Delta L(i))$ per unit length [6], [12]. These parameters can be related to HTS intrinsic nonlinearities as follows: the nonlinear resistance $\Delta R$ is due to nonlinearities in quasi-particle conductivity and the nonlinear inductance $\Delta L(i)$ is due to nonlinearities in penetration depth. At sufficiently small currents, we should expect a quadratic dependence of the overall inductance and resistance per unit length on the total current $i$ through the cross section of the TL

$$
\begin{aligned}
& R(i)=R_{d}+\Delta R(i) \text { with } \Delta R(i)=\Delta R_{q} \cdot i^{2} \\
& L(i)=L_{d}+\Delta L(i) \text { with } \Delta L(i)=\Delta L_{q} \cdot i^{2}
\end{aligned}
$$

where $\Delta L_{q}$ and $\Delta R_{q}$ are positive to be consistent with the model of intrinsic nonlinearities in HTS TLs [11], [19]. These nonlinear terms just add to the linear $R_{d}, L_{d}, C_{d}$, and $G_{d}$ distributed elements of the model of a TL (Fig. 1).

For larger signals and close to the transition temperature, intrinsic nonlinearities increase drastically, thus (1) and (2) would have to include higher order terms or even be written in forms other than a Taylor's series. Besides, as mentioned earlier, extrinsic HTS nonlinearities may also give rise to nonlinearities in $R(i)$ and $L(i)$, but their mathematical treatment is not different from the one of (1) and (2) so the distinction between intrinsic and extrinsic HTS nonlinearities will not be made henceforth.

\section{B. Nonlinearities in Ferroelectric Oxides}

Ferroelectric oxides also exhibit large microwave nonlinear effects due to a dependence of the dielectric constant $\left(\varepsilon_{r}\right)$ on the electric field (or voltage $v$ ) inside the ferroelectric. The dielectric nonlinearity $\varepsilon_{r}(v)$ is an intrinsic property of ferroelectrics in their paraelectric phase, whichever the type of ferroelectrics (incipient or displacive). It relates the polarization state of the material with the applied voltage or electric field. This potential variation $\varepsilon_{r}(v)$ is interesting to control microwave devices [3], [20] via a direct external voltage, but also produces nonlinearities. As the microwave input power is increased in a device, the electric field strength increases, changing the value of $\varepsilon_{r}$ in a nonlinear form. To model the nonlinear dependence of $\varepsilon_{r}$ in the case of a microwave circuit, a nonlinear capacitance $\Delta C(v)$ and a nonlinear conductance $\Delta G(v)$ are introduced [21]. A quadratic model is also chosen to fit the IMD or 3rdH data - as most of the experimental data suggest [22] —as follows:

$$
\begin{aligned}
& C(v)=C_{d}+\Delta C(v) \text { with } \Delta C(v)=\Delta C_{q} \cdot v^{2} \\
& G(v)=G_{d}+\Delta G(v) \text { with } \Delta G(v)=\Delta G_{q} \cdot v^{2} .
\end{aligned}
$$

Nevertheless, other dependencies have also been reported and explained [23]. As previously done for HTS, these nonlinear components will be added to $R_{d}, L_{d}, C_{d}$, and $G_{d}$ of the distributed model of a TL (Fig. 1). It should be noted that $\Delta C_{q}$ is negative as opposed to $\Delta L_{q}$ [24]. As discussed in Section IV, this could make possible the compensation of nonlinear effects between ferroelectrics and HTS.

\section{IMD AND THIRD HARMONIC IN TLs}

\section{A. Closed-Form Expressions for IMD and $3 \mathrm{rdH}$}

Fig. 1 shows the equivalent circuit of an infinitesimal segment of TL (of length $d z$ ) showing the nonlinear contributions of HTS and ferroelectric according to (1)-(4). This simple, but representative model will be used to extract closed-form expressions of the third-order intermodulation product and harmonic signals generated in matched TLs or TL resonators. These structures are the basis of many microwave circuits and they allow a fundamental investigation with direct repercussions on applications.

From the linear model of a TL given in Fig. 1, the telegraphers' equations can be written as follows:

$$
\begin{aligned}
& \frac{\partial i}{\partial z}=-\frac{\partial\left(C_{d} v\right)}{\partial t}-G_{d} v \\
& \frac{\partial v}{\partial z}=-\frac{\partial\left(L_{d} i\right)}{\partial t}-R_{d} i .
\end{aligned}
$$

If nonlinear terms are introduced, they will generate spurious signals such as higher order harmonics or IMD due to mixing of different frequencies. In the following, the subscript $i$ is used to denote the different frequencies of interest $f_{i}$. Individual tone at $f_{0}$ or two closely separated tones at $f_{1}$ and $f_{2}$ are applied to get the third harmonic at $f_{3}=3 f_{0}$ or the IMD product at $f_{12}=2 f_{1}-f_{2}$, respectively.

To model the nonlinear TL, (1)-(4) are introduced in the telegraphers' equations (5) and (6). The resulting equations (7) 
and (8) describe a TL containing a nonlinear current generator $\left(\partial i_{\mathrm{nl}} / \partial z\right)$ and a nonlinear voltage generator $\left(\partial v_{\mathrm{nl}} / \partial z\right)$

$$
\begin{aligned}
& \frac{\partial i}{\partial z}=-C_{d} \frac{\partial v}{\partial t}-G_{d} v-\frac{\partial i_{n l}}{\partial z} \\
& \frac{\partial v}{\partial z}=-L_{d} \frac{\partial i}{\partial t}-R_{d} i-\frac{\partial v_{n l}}{\partial z}
\end{aligned}
$$

with the nonlinear contributions

$$
\begin{aligned}
& \frac{\partial v_{n l}}{\partial z}=\frac{\partial[\Delta L(i) \cdot i]}{\partial t}+\Delta R(i) \cdot i \\
& \frac{\partial i_{n l}}{\partial z}=\frac{\partial[\Delta C(v) \cdot v]}{\partial t}+\Delta G(v) \cdot v .
\end{aligned}
$$

The combination of (7) and (8) with (1)-(4) results in a propagation equation written in the frequency domain for a given frequency $\omega_{i}$

$$
\frac{\partial^{2} I_{i}}{\partial z^{2}}=\gamma_{i}^{2} I_{i}+\frac{\gamma_{i}}{Z_{0, i}}\left(\frac{\partial V_{n l, i}}{\partial z}\right)-\frac{\partial^{2} I_{n l, i}}{\partial z^{2}}
$$

where $\gamma_{i}$ is the propagation constant and $Z_{0, i}$ is the characteristic impedance at $\omega_{i}$

$$
\begin{aligned}
\gamma_{i} & =\sqrt{\left(G_{d, i}+j \omega_{i} C_{d}\right)\left(R_{d, i}+j \omega_{i} L_{d}\right)}=\alpha_{i}+j \beta_{i} \\
Z_{0, i} & =\sqrt{\frac{R_{d, i}+j \omega_{i} L_{d}}{G_{d, i}+j \omega_{i} C_{d}}} .
\end{aligned}
$$

The nonlinear current generator $\left(\partial i_{\mathrm{nl}} / \partial z\right)$ and the nonlinear voltage generator $\left(\partial v_{\mathrm{nl}} / \partial z\right)$ take the following general forms in the frame of quadratic nonlinearities (1)-(4):

$$
\begin{aligned}
\frac{\partial I_{n l, i}}{\partial z} & =A_{\mathrm{FER}, i} F\left(v^{3}(z, t), \omega_{i}\right) \\
\frac{\partial V_{n l, i}}{\partial z} & =A_{\mathrm{HTS}, i} F\left(i^{3}(z, t), \omega_{i}\right)
\end{aligned}
$$

where $A_{\mathrm{HTS}, i}=\left(\Delta R_{q}+j \omega_{i} \Delta L_{q}\right), A_{\mathrm{FER}, i}=\left(\Delta G_{q}+\right.$ $\left.j \omega_{i} \Delta C_{q}\right)$, and $F\left(v^{3}(z, t), \omega_{i}\right)$ is the Fourier transform of $v^{3}(z, t)$ particularized at $\omega_{i}$. This function depends on the voltage distribution in the line at $\omega_{i}$. Likewise, $F\left(i^{3}(z, t), \omega_{i}\right)$ is the Fourier transform of $i^{3}(z, t)$ particularized at $\omega_{i}$ and depends on the current distribution in the line at $\omega_{i}$. The following paragraphs detail $F\left(v^{3}(z, t), \omega_{i}\right)$ and $F\left(i^{3}(z, t), \omega_{i}\right)$ for the particular cases of a traveling-wave TL and a resonant TL.

1) Traveling Wave Along a Matched TL: The expression of the current (voltage) of a traveling wave along a matched TL can take the following form in the time domain:

$$
i_{i}(z, t)=\operatorname{Re}\left[I_{i}(z) e^{j \omega_{i} t}\right]
$$

with

$$
I_{i}(z)=\left|I_{i}\right| e^{j \phi_{i}} e^{-\left(\alpha_{i}+j \beta_{i}\right) z} .
$$

The substitution of (16) and (17) and their equivalent expressions for the voltage $\left(v_{i}(z, t)\right.$ and $\left.V_{i}(z, t)\right)$ into (14) and (15) of the nonlinear generators yields the expression for the functions $F\left(i^{3}(z, t), \omega_{i}\right)$ and $F\left(\nu^{3}(z, t), \omega_{i}\right)$ at the IMD frequency $\omega_{12}$

$$
\begin{aligned}
& \frac{\partial I_{n l, 12}}{\partial z}=\frac{3}{4} V_{1}^{2} V_{2}^{*} A_{\mathrm{FER}, 12} e^{-2 \alpha_{1} z} e^{-\gamma_{1} z} \\
& \frac{\partial V_{n l, 12}}{\partial z}=\frac{3}{4} I_{1}^{2} I_{2}^{*} A_{\mathrm{HTS}, 12} e^{-2 \alpha_{1} z} e^{-\gamma_{1} z}
\end{aligned}
$$

and for the third-order harmonic

$$
\begin{aligned}
\frac{\partial I_{n l, 3}}{\partial z} & =\frac{1}{4} V_{1}^{3} A_{\mathrm{FER}, 3} e^{-3 \gamma_{1} z} \\
\frac{\partial V_{n l, 3}}{\partial z} & =\frac{1}{4} I_{1}^{3} A_{\mathrm{HTS}, 3} e^{-3 \gamma_{1} z} .
\end{aligned}
$$

As done in [6] for a matched superconducting TL, we can assume that the propagation equation (11) accepts solutions of the form

$$
\begin{aligned}
I_{12}(z) & =K_{12}(z) e^{-\gamma_{12} z} \\
I_{3}(z) & =K_{3}(z) e^{-\gamma_{3} z}
\end{aligned}
$$

for the IMD and 3rdH, respectively. $K_{12}(0)=0$ and $K_{3}(0)=$ 0 . Using (18) and (19) to solve (11) for a traveling-wave TL in a similar way, as done in [6], we obtain the following expression for the IMD current as a function of the distance from the source $z$ :

$$
\begin{aligned}
I_{12}(z)=\frac{3}{16} \frac{I_{1}^{2} I_{2}^{*}}{Z_{0,1}}[ & \gamma_{1} A_{\mathrm{HTS}, 12}+\left(2 \alpha_{1}+\gamma_{1}\right) \\
& \left.\times Z_{0,1}^{2}\left|Z_{0,1}\right|^{2} A_{\mathrm{FER}, 12}\right] \frac{e^{-2 \alpha_{1} z}-1}{\alpha_{1}\left(\alpha_{1}+\gamma_{1}\right)} e^{-\gamma_{1} z}
\end{aligned}
$$

where, to simplify this expression, we have assumed that both fundamental frequencies are very close to each other so propagation constants and characteristic impedances are similar: $\gamma_{12} \approx \gamma_{2} \approx \gamma_{1}$ and $Z_{0,12} \approx Z_{0,2} \approx Z_{0,1}$.

Equation (24) can be further simplified if we assume low propagation loss $e^{-2 \alpha_{1} z} \approx 1-2 \alpha_{1} z$ and $2 \alpha_{1}+\gamma_{1} \approx \gamma_{1}$ as follows:

$$
I_{12}(z)=-\frac{3}{8} \frac{I_{1}^{2} I_{2}^{*}}{Z_{0,1}}\left[A_{\mathrm{HTS}, 12}+Z_{0,1}^{2}\left|Z_{0,1}\right|^{2} A_{\mathrm{FER}, 12}\right] z e^{-\gamma_{1} z} .
$$

The IMD power delivered to a terminated load located at a distance $l$ from the source can be easily calculated according to $P_{12}=Z_{0,1}\left|I_{12}(l)\right|^{2} / 2$

$$
P_{12}=\left.\left.\frac{9 l^{2}}{16 Z_{0,1}^{4}}\left|A_{\mathrm{HTS}, 12}+Z_{0,1}^{2}\right| Z_{0,1}\right|^{2} A_{\mathrm{FER}, 12}\right|^{2} P_{1}^{2} P_{2} .
$$

For the $3 \mathrm{rdH}$, the procedure is similar and the approximation $3 \beta_{1} \equiv \beta_{3}$ will be used as the ferroelectrics are known to be nondispersive until frequencies of the order of $10^{11}-10^{12} \mathrm{~Hz}$ 
[24], but note that, in this case, $3 \alpha_{1} \neq \alpha_{3}$ and, thus, $3 \gamma_{1} \neq \gamma_{3}$. The result is

$$
\begin{aligned}
I_{3}=\frac{I_{1}^{3}}{4}\left[\frac{\gamma_{3}}{Z_{0,3}} A_{\mathrm{HTS}, 3}+\right. & \left.3 \gamma_{1} Z_{0,1}^{3} A_{\mathrm{FER}, 3}\right] \\
& \times \frac{\left(e^{-\left(3 \gamma_{1}-\gamma_{3}\right) z}-1\right) e^{-\gamma_{3} z}}{\left(3 \gamma_{1}-\gamma_{3}\right)\left(3 \gamma_{1}+\gamma_{3}\right)} .
\end{aligned}
$$

For small dispersion and weak attenuation, $3 \gamma_{1} \approx \gamma_{3}$ and the simplified expression of the $3 \mathrm{rdH}$ output power will be

$$
P_{3}=\frac{l^{2}}{16 Z_{0,1}^{3} Z_{0,3}}\left|A_{\mathrm{HTS}, 3}+Z_{0,1}^{3} Z_{0,3} A_{\mathrm{FER}, 3}\right|^{2} P_{1}^{3} .
$$

Equations (24)-(28) show that the signals generated by the ferroelectric nonlinearities are superimposed to those caused by the nonlinearities in the HTS.

2) Resonant TL: Considering the resonant mode at $l=\lambda / 2$ of an open-ended TL, the current and voltage along the resonator can be written as

$$
\begin{aligned}
& i_{i}(z, t)=\operatorname{Re}\left[I_{i} e^{j \omega_{i} t} \sin \left(\frac{\pi z}{l}\right)\right] \\
& v_{i}(z, t)=\operatorname{Re}\left[V_{i} e^{j \omega_{i} t} \sin \left(\frac{\pi z}{l}+\frac{\pi}{2}\right)\right]
\end{aligned}
$$

where the maximum voltage $V_{i}$ and current $I_{i}$ in the line are related by

$$
V_{i}=j Z_{0, i} I_{i} .
$$

Substitution of (29) and (30) into (14) and (15) yields an expression for the nonlinear terms due to the ferroelectric layer and the HTS film

$$
\begin{aligned}
\frac{\partial I_{n l, 12}}{\partial z} & =\frac{3}{4} V_{1}^{2} V_{2}^{*} A_{\mathrm{FER}, 12} \sin ^{3}\left(\frac{\pi z}{l}+\frac{\pi}{2}\right) \\
\frac{\partial V_{n l, 12}}{\partial z} & =\frac{3}{4} I_{1}^{2} I_{2}^{*} A_{\mathrm{HTS}, 12} \sin ^{3}\left(\frac{\pi z}{l}\right) .
\end{aligned}
$$

The expression of the peak IMD current at $l / 2$ can be obtained by equating the power dissipated into the resonator at $\omega_{12}$ with the power generated also at $\omega_{12}$ [6] as follows:

$$
-\frac{1}{2} \operatorname{Re}\left[\int_{0}^{l}\left(V_{12} \partial I_{n l}^{*}+\partial V_{n l} I_{12}^{*}\right) d z\right]=\frac{\omega_{12}}{2 Q_{L}} \int_{0}^{l} L_{d}\left|I_{12}(z)\right|^{2} d z
$$

so that, combining (29)-(34), we obtain

$$
I_{12}=-\frac{9}{16} \frac{Q_{L}}{\omega_{12} L_{d}} I_{1}^{2} I_{2}^{*}\left[A_{\mathrm{HTS}, 12}+\left|Z_{0,1}\right|^{2} Z_{0,1}^{2} A_{\mathrm{FER}, 12}\right] .
$$

Note that in (35), the term into brackets, which quantifies the nonlinear behavior, is the same as in (25). The output power dissipated in a terminal load located at a distance $l$ from the source and accessible experimentally can be calculated using conventional microwave theory [25] as follows:

$$
P_{12}=\frac{1}{4} \beta l\left(R_{d, 12}\left|I_{12}\right|^{2}+G_{d, 12}\left|Z_{0,12}\right|^{2}\left|I_{12}\right|^{2}\right) .
$$

In (36), $\beta$ is the output coupling factor that quantifies the fraction of power flowing from the resonator to the load impedance [26].
In (24)-(28) and (35), the particular condition $A_{\mathrm{HTS}, i}=0$ represents a device where the conductor is linear. This would be the case if a metal is used instead of a superconductor. The equations then describe ferroelectric nonlinearities alone. Similarly, $A_{\mathrm{FER}, i}=0$ describes conducting (e.g., HTS) nonlinearities alone, as the nonlinear source from the substrate would not be active. The latter situation is described in [6] and the equations derived there agree with (24)-(28) and (35) taking $A_{\mathrm{FER}, i}=0$.

\section{B. Validation of the Closed-Form Expressions by Comparison With Harmonic-Balance Simulations}

The proposed closed-form expressions are very useful, but present limitations, as they do not consider compression effects or higher order nonlinear effects that could also influence both the fundamental and third-order terms if the circulating power in the device is very high.

In order to identify the limit of validity of the equations, we have done HB simulations. This method was previously used to model the nonlinearities from HTS and a detailed description on how it is applied for distributed nonlinearities can be found in [6]. Simulations based on the HB algorithm provide a large flexibility on the device design [27] and type of input signals [28]. The TL section shown in Fig. 1 is the elemental cell of length $d z$. The distributed nature of the electrical parameters is taken into account by cascading a large number of such elemental cells $(n=l / d z)$ until it matches the length of the line.

1) Matched TL: The simulation is done for a traveling-wave propagating in a coplanar TL of length $47.07 \mathrm{~mm}$ and characteristic impedance $Z_{0}=50 \Omega$. The width of the central line is $50 \mu \mathrm{m}$ and the gap between the central line and ground planes is $100 \mu \mathrm{m}$. This line is made of a $0.508-\mathrm{mm}$ thick $\mathrm{LaA}_{1} \mathrm{O}_{3}$ substrate $\left(\varepsilon_{r}=24, \tan \delta=10^{-6}\right)$ that has a ferroelectric layer $\left(\varepsilon_{r}=500, \tan \delta=10^{-3}\right)$ and superconducting electrodes $\left(R_{s}=40 \mu \Omega\right.$ at $5 \mathrm{GHz}$ ). The effective $\tan \delta=10^{-4}$ is estimated from computation of the filling factors and effective dielectric constant [29]. Computation of IMD is done by applying two tones at $f_{1}=5 \mathrm{GHz}$ and $f_{2}=5.0001 \mathrm{GHz}$. The $3 \mathrm{rdH}$ is computed for a single input tone at $f_{1}=5 \mathrm{GHz}$ and we look at the output at $f_{3}=15 \mathrm{GHz}$. The line is divided in 200 segments per wavelength representing a total of $n=600$ cells.

The values of the distributed linear elements $R_{d}, L_{d}, C_{d}$, and $G_{d}$ were calculated from the line dimensions using MATRIX. The distributed nonlinear terms $\Delta R_{q}$ and $\Delta L_{q}$ are obtained from Sheen's model for superconductors [30] and $\Delta R_{q}=33.85 \Omega /\left(\mathrm{mA}^{2}\right), \Delta L_{q}=1.075510^{-9} \mathrm{H} /\left(\mathrm{mA}^{2}\right)$.

With the sole purpose of checking the validity of (24)-(28), we choose $\Delta G_{q}=2 \Delta R_{q} / Z_{0}^{4}=8.610^{-6} 1 /\left(\Omega \cdot \mathrm{mV}^{2}\right)$ and $\Delta C_{q}=-2 \Delta L_{q} / Z_{0}^{4}=-2.710^{-16} \mathrm{~F} /\left(\mathrm{mV}^{2}\right)$.

Fig. 2 shows that the closed-form expressions fit the results of the HB simulation on a large range of input power for a traveling-wave TL. The absolute error before compression effects occur is less than $2 \%$.

2) Resonant TL: The resonator model is an open-ended coplanar TL of length $l=7.8 \mathrm{~mm}$ with capacitive gap couplings to the source and load, otherwise with a similar cross section than the traveling-wave TL. This geometry yields a resonance at $f_{0} \approx 5.32 \mathrm{GHz}$. We again choose $\Delta G_{q}=2 \Delta R_{q} / Z_{0}^{4}$, $\Delta C_{q}=-2 \Delta L_{q} / Z_{0}^{4}$. 


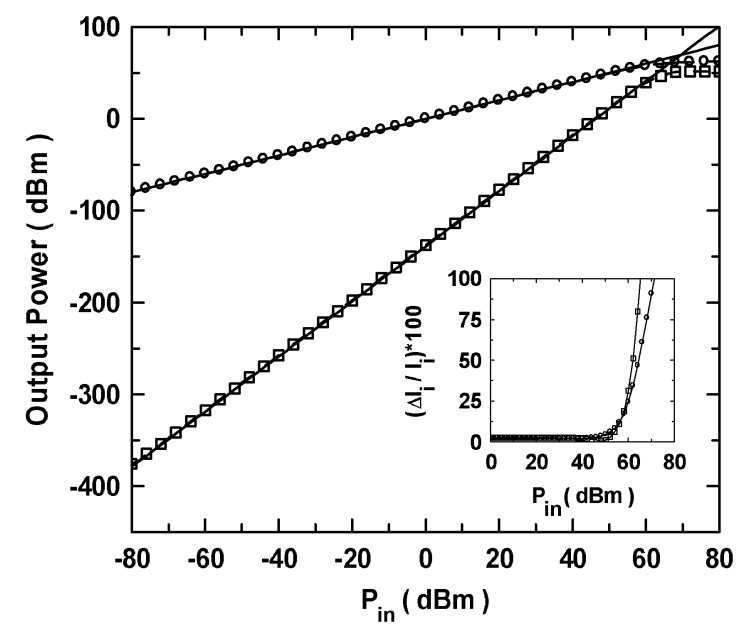

Fig. 2. Comparison of the power of the fundamental and third-order harmonic calculated from closed-form expressions (28) (straight lines) and HB method (circles and squares) as a function of the incident microwave power $P_{\text {in }}$ for a traveling wave propagating in a TL made of a dielectric substrate $\left(\mathrm{LaAlO}_{3}\right)$, a ferroelectric layer, and HTS electrodes. The inset shows the absolute error vesus input power for the fundamental and third harmonic. Similar results are found for the IMD.

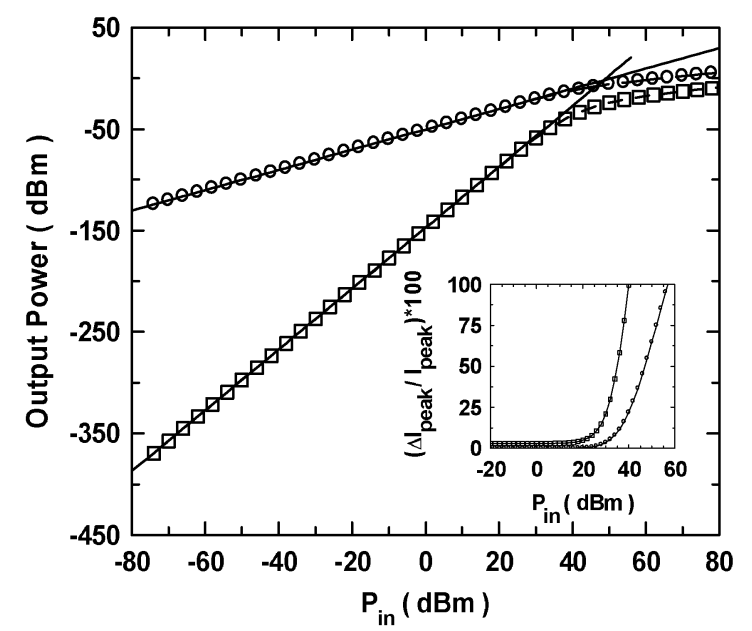

Fig. 3. Comparison of the IMD calculated from closed-form expressions (34)-(36) (straight lines) and HB method (circles) as a function of the incident microwave power $P_{\text {in }}$ for a resonant TL integrating a ferroelectric layer and HTS electrodes. The inset gives the absolute error on the fundamental and IMD peak currents.

As observed on Fig. 3, (35) and (36) are well suited to fit the results from HB simulation, although compression effects rapidly occur due to large peak currents in high- $Q$ resonators. The compression effects limit the validity of the closed-form expressions. The inset of Fig. 3 gives the absolute error between the peak currents obtained with both methods for the fundamental and IMD. In the presented case of an undercoupled resonator $\left(S_{12} \approx-20 \mathrm{~dB}\right)$, the distribution of the signal in the line closely follows (29) and (30) and the absolute error is less than $1 \%$. A deviation from (29) and (30) may happen for strongly coupled resonators. In the case where $S_{12} \approx-5 \mathrm{~dB}$, the absolute error is slightly less than $10 \%$.

\section{COMPENSATION BETWEEN NONLINEAR EFFECTS}

According to the equations derived thus far, it is theoretically possible to compensate the IMD and 3rdH generated by the nonlinearities in the dielectric with those generated by the superconductor. The IMD and 3rdH could cancel under the following conditions, respectively:

$$
\begin{aligned}
A_{\text {HTS.12 }} & =Z_{1,0}^{2}\left|Z_{0,1}\right|^{2} A_{\text {FER. } 12} \\
A_{\text {HTS.3 }} & =Z_{1,0}^{3} Z_{0,3} A_{\text {FER.3. }} .
\end{aligned}
$$

Note that (37) and (38) are the same condition if $Z_{0,1} \approx Z_{0,3}$. Note also that (37) is the condition to cancel the IMD signal in a TL resonator. IMD and $3 \mathrm{rdH}$ signals cancel for

$$
\Delta R_{q}=-\left|Z_{0,1}^{2}\right|^{2} \Delta G_{q} \text { and } \Delta L_{q}=-\left|Z_{0,1}^{2}\right|^{2} \Delta C_{q} .
$$

The conditions posed by (37)-(39) are valid for any type of NLTL and may hold over a broad band of frequencies if the characteristic impedance can be assumed to be constant and real. These equations have been tested with HB simulations and a significant decrease of the nonlinear response was observed when both sources of nonlinearities are present under the conditions of (37)-(39). As shown, the compensation is dependent on the magnitude and the sign of all nonlinear terms $\Delta R_{q}, \Delta L_{q}, \Delta C_{q}$, and $\Delta G_{q}$.

For the specific case of NLTLs with HTS and ferroelectrics, full cancellation of the real part is not possible since $\Delta R_{q}$ and $\Delta G_{q}$ have the same sign and, therefore, (39) cannot be strictly fulfilled. Note, however, that this is not the case for $\Delta L_{q}$, $\Delta C_{q}$, thus almost full (and broad-band) cancellation would be possible if reactive nonlinearities dominate over the resistive ones. For this to occur, one should use ferroelectric layers with values of $\Delta C_{q}$ much smaller than those usually reported in the literature. For example, $\Delta C$ values in [22] are approximately 20 times larger than the $\Delta C_{q}$ value used in this paper. These values might be reduced by acting on the thickness of the ferroelectric layer, on the dimensions of the layout of the CPW line, or on the dc bias of the ferroelectric layer.

Finally, we would like to note that, although we have focused on the compensation of IMD and 3rdH in NLTLs, the scope of application of these results is broad since there are many situations where the nonlinear performance of a complex device may be modeled as several NLTLs, e.g., in the case of a planar HTS filter [27].

\section{CONCLUSION}

This paper proposes a description of nonlinear effects from different origins and likely to occur in materials to be integrated in novel high-performance microwave devices. The model integrates linear and nonlinear distributed parameters to represent the distributed nature of the nonlinearities in a TL. It is a deeper development of a previous model written for HTS nonlinearities only that has proven its validity [6]. This new model gives closed-form expressions (24)-(28) and (34)-(36) for the IMD and $3 \mathrm{rdH}$ signals in the case of simple devices made with TLs, when conducting nonlinearities (from the HTS) and dielectric nonlinearities (from the ferroelectric) are combined. Its extension to disk resonators was previously analyzed for HTS nonlinearities [5], [31] and would not present more difficulties if 
two types of nonlinearities were active. Equations (24)-(28) and (36) were subsequently validated through comparison with the results of $\mathrm{HB}$ simulations.

In the framework of this modeling, we considered that each type of nonlinearity could be isolated, which should not be easy from microwave measurement of a multilayer. The presence of one layer may indeed influence the nonlinearities of the other (e.g., because of interface issues). Under these conditions, it was observed that the nonlinear effects just cumulate in the case of a traveling wave and for a resonant open-ended TL. The important result observed in this paper is that it is theoretically possible to reduce the nonlinear contribution of the HTS by using a thin ferroelectric layer. More generally, nonlinear effects of different origins could compensate between them. Conditions to cancel the IMD and 3rdH signal have been extracted for NLTLs. This could, for example, be a way to solve the limitations of IMD occurring in state-of-the-art planar HTS filters.

\section{ACKNOWLEDGMENT}

The authors would like to thanks Dr. J. Booth, National Institute of Standards and Technology (NIST), Boulder, CO, for fruitful discussion on the nonlinearities in the ferroelectrics and its modeling.

\section{REFERENCES}

[1] O. G. Vendik, E. K. Hollmann, A. B. Kozyrev, and A. M. Prudan, "Ferroelectric tuning of planar and bulk microwave devices," J. Supercond., vol. 12, no. 2, pp. 325-338, 1999.

[2] B. H. Moeckly and Y. Zhang, "Strontium titanate thin films for tunable $\mathrm{YBa}_{2} \mathrm{Cu}_{3} \mathrm{O}_{7}$ microwave filters," IEEE Trans. Appl. Supercond., vol. 11, no. 1 , pp. 450-453, Mar. 2001.

[3] B. Marcilhac, D. G. Crété, Y. Lemaître, D. Mansart, J. C. Mage, K. Bouzehouane, C. Dolin, E. Jacquet, P. Woodall, and J. P. Contour, "Frequency-agile microwave devices based on $\mathrm{Y}-\mathrm{Ba}-\mathrm{Cu}-\mathrm{O} / \mathrm{Sr}-\mathrm{Ti}-\mathrm{O} / / \mathrm{LaA}_{1} \mathrm{O}_{3}$ structure," IEEE Trans. Appl. Supercond., vol. 11, no. 1, pp. 438-441, Mar. 2001.

[4] J. C. Booth, L. R. Vale, R. H. Ono, and J. H. Claassen, "Power-dependent impedance of high temperature thin films: Relation to harmonic generation," J. Supercond., vol. 89, no. 12, pp. 7750-7755, Dec. 2001.

[5] D. E. Oates, P. P. Nguyen, G. Dresselhaus, M. S. Dresselhaus, G. Koren, and E. Polturak, "Nonlinear surface impedance of YBCO thin films: Measurements, modeling and effects in devices," J. Supercond., vol. 8, no. 6, pp. 725-733, 1995

[6] C. Collado, J. Mateu, and J. O'Callaghan, "Analysis and simulation of the effects of distributed nonlinearities in microwave superconducting devices," IEEE Trans. Appl. Supercond., vol. 15, no. 1, pp. 26-39, Mar. 2005

[7] K. S. Champlin and D. R. Singh, "Small-signal second-harmonic generation by a nonlinear transmission line," IEEE Trans. Microw. Theory Tech., vol. 34, no. 3, pp. 351-353, Mar. 1986.

[8] O. G. Vendik and S. P. Zubko, "Ferroelectric phase transition and maximum dielectric permittivity of displacement type ferroelectrics $\left(\mathrm{Ba}_{x} \mathrm{Sr}_{1-x} \mathrm{TiO}_{3}\right)$," J. Appl. Phys., vol. 88, no. 9, pp. 5343-5350, 2000.

[9] - "Modeling the dielectric response of incipient ferroelectrics," $J$. Appl. Phys., vol. 82, no. 9, pp. 4475-4483, 1997.

[10] M. H. Hein, D. E. Oates, P. J. Hirst, R. G. Humphreys, and A. V. Velichko, "Nonlinear dielectric microwave losses in MgO substrates," Appl. Phys. Lett., vol. 80, no. 6, pp. 1007-1009, 2002.

[11] D. Xu, S. K. Yip, and J. A. Sauls, "Nonlinear Meissner effect in unconventionnal superconductors," Phys. Rev. B, Condens. Matter, vol. 51, no. 22, pp. 16233-16253, 1995.

[12] T. Dahm and D. J. Scalapino, "Theory of intermodulation in a superconducting microstrip resonator," J. Appl. Phys, vol. 81, no. 4, pp. 2002-2008, 1995 .

[13] M. Hein, W. Diete, M. Getta, S. Hensen, T. Kaiser, G. Müller, H. Piel, and H. Schlick, "Fundamental limits of the linear microwave power response of epitaxial Y-Ba-Cu-O films," IEEE Trans. Appl. Supercond., vol. 7, no. 2, pp. 1264-1267, Jun. 1997.
[14] J. Halbritter, "Extrinsic or intrinsic conduction in cuprates: Anisotropy, weak and strong links," Phys. Rev. B, Condens. Matter, vol. 48, no. 13 , pp. 9735-9746, 1993.

[15] D. E. Oates, M. A. Hein, P. J. Hirst, R. G. Humphreys, G. Koren, and E. Polturak, "Nonlinear microwave surface impedance of YBCO films: Latest results and present understanding," Physica C, vol. 372-376, pp. 462-468, 2002.

[16] W. Diete, M. Getta, M. Hein, T. Kaiser, G. Müller, H. Piel, and H. Schlick, "Surface resistance and nonlinear dynamic microwave losses of epitaxial HTS films," IEEE Trans. Appl. Supercond., vol. 7, no. 2, pp. 1236-1239, Jun. 1997.

[17] D. E. Oates, S.-H. Park, and G. Koren, "Observation of the nonlinear Meissner effect in YBCO thin films: Evidence for a $d$-wave order parameter in the bulk of the cuprate superconductors," Phys. Rev. Lett., vol. 93, no. 19, pp. 197 001/1-197001/4, 2004.

[18] B. A. Willemsen, K. E. Kihlstrom, and T. Dahm, "Unusual power dependence of two-tone intermodulation in high- $T_{c}$ superconducting microwave resonators," Appl. Phys. Lett., vol. 74, no. 5, pp. 753-755, 1999.

[19] P. P. Nguyen, D. E. Oates, G. Dresselhaus, M. S. Dresselhaus, and A. C. Anderson, "Microwave hysteretic losses in $\mathrm{YBa}_{2} \mathrm{Cu}_{3} \mathrm{O}_{7-x}$ and NbN thin films," Phys. Rev. B, Condens. Matter, vol. 51, no. 10, pp. 6686-6695, 1995

[20] M. J. Lancaster, J. Powell, and A. Porch, "Thin-film ferroelectric microwave devices," Supercond. Sci. Technol., vol. 11, pp. 1323-1334, 1998.

[21] A. B. Kozyrev et al., "Nonlinear behavior of thin film $\mathrm{SrTiO}_{3}$ capacitors frequencies," J. Appl. Phys., vol. 84, no. 6, pp. 3326-3332, 1998.

[22] J. C. Booth, R. H. Ono, I. Takeuchi, and K.-S. Chang, "Microwave frequency tuning and harmonic generation in ferroelectric thin film transmission lines," Appl. Phys. Lett., vol. 81, no. 4, pp. 718-720, 2002.

[23] R. Ott, P. Lahl, and R. Wördenweber, "Nonlinear microwave properties of ferroelectric thin films," Appl. Phys. Lett., vol. 84, no. 21, pp. 4147-4149, 2004.

[24] A. Kozyrev, A. Ivanov, T. Samoilova, O. Soldatenkov, K. Astafiev, and L. C. Sengupta, "Nonlinear response and power handling capability of ferroelectric $\mathrm{Ba}_{x} \mathrm{Sr}_{1-x} \mathrm{TiO}_{3}$ film capacitors and tunable microwave devices," J. Appl. Phys., vol. 88, no. 9, pp. 5334-5342, 2000.

[25] D. M. Pozar, Microwave Engineering. New York: Wiley, 1998.

[26] M. J. Lancaster, Passive Microwave Devices Applications of High Temperature Superconductors. Cambridge, U.K.: Cambridge Univ. Press, 1997.

[27] J. Mateu, O. Menédez, C. Collado, and J. M. O'Callaghan, "Nonlinear performance characterization in an eight-pole quasi-elliptic bandpass filter," Supercond. Sci. Technol., vol. 17, no. 5, pp. S359-S362, 2004.

[28] C. Collado, J. Mateu, R. Ferrus, and J. M. O'Callaghan, "Prediction of nonlinear distortion in HTS filters for CDMA communications systems," IEEE Trans. Appl. Supercond., vol. 13, no. 2, pp. 328-331, Jun. 2003.

[29] H.-T. Lue and T.-Y. Tseng, "Application of on-wafer TRL calibration on the measurement of microwave properties of $\mathrm{Ba}_{0.5} \mathrm{Sr}_{0.5} \mathrm{TiO}_{3}$ thin films," IEEE Trans. Ultrason., Ferroelect., Freq. Control, vol. 48, no. 6, pp. 1640-1647, Nov. 2001.

[30] D. M. Sheen, S. M. Ali, D. E. Oates, R. S. Withers, and J. A. Kong, "Current distribution, resistance, and inductance for superconducting strip transmission lines," IEEE Trans. Appl. Supercond., vol. 1, no. 2, pp. 108-115, Jun. 1991.

[31] J. Mateu, C. Collado, and J. M. O'Callaghan, "Nonlinear analysis of disk resonators: Application to material characterization and filter design," IEEE Trans. Appl. Supercond., vol. 11, no. 1, pp. 135-138, Mar. 2001.

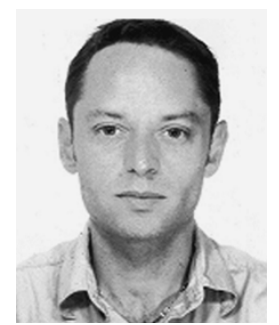

David Seron was born in Caen, France, in 1974. He received the Master degree in materials sciences and $\mathrm{Ph} . \mathrm{D}$. degree in physics from the Université Paris VI, Pierre \& Marie Curie, Paris, France, in 1998 and 2002, respectively.

From 2002 to 2003, in collaboration with the Analog Devices Group, Massachusetts Institute of Technology (MIT) Lincoln Laboratory, he was a Post-Doctoral Fellow with the Research Laboratory of Electronics, MIT. In 2004, he was a Research Associate with the Institute of Material Science of Barcelona (ICMAB). At the end of 2004, he joined the Universitat Politècnica de Catalunya (UPC), Barcelona, Spain. His research interests include the high-frequency characterization of materials with an emphasis on high- $T c$ superconductors and ferroelectric and ferromagnetic materials. 


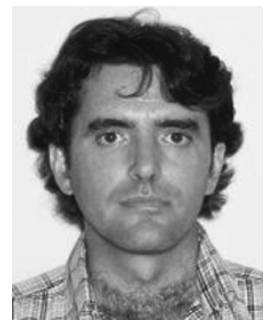

Carlos Collado (M'05) was born in Barcelona, Spain, in 1969. He received the Telecommunication Engineering degree and Ph.D. degree from the Universitat Politècnica de Catalunya (UPC), Barcelona, Spain, in 1995 and 2001, respectively, and the M.S. degree in bioengineering from the Biomedical Engineering Research Centre, UPC, in 2002.

In 1998, he joined the faculty of UPC, where he has been teaching courses on theory of electromagnetism, microwave laboratory, and high-frequency devices and systems. From June to December 2004 he was a Visiting Researcher with the University of California at Irvine. Since April 2005, he has been an Associate Professor with UPC. His primary research interests include microwave devices and systems, electrooptics applications, and superconducting devices.

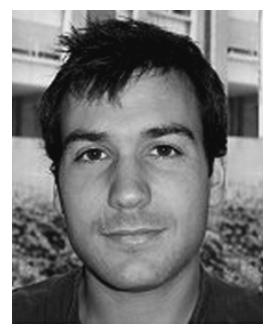

Jordi Mateu (M'05) was born in Llardecans, Spain in 1975. He received the Telecommunication Engineering and Ph.D. degrees from the Universitat Politècnica de Catalunya (UPC), Barcelona, Spain, in 1999 and 2003, respectively.

Since September 2005, he has been a Fulbright Research Fellow with the National Institute of Standards and Technology (NIST), Boulder, CO. From September 1999 to September 2002, he was Research Assistant with the Department of Signal Theory and Communications, UPC. From May to August 2002, he as a Visiting Researcher with Superconductor Technologies Inc., Santa Barbara, CA. From October 2002 to August 2005, he was Research Associate with the Telecommunications Technological Center of Catalonia (CTTC), Barcelona, Spain. During September 2004, he was Visiting Researcher with NIST. From September 2003 to August 2005, he was Part-Time Assistant Professor with the Universitat Autònoma de Barcelona. His primary research interests include microwave and photonic devices and systems and superconducting devices with special emphasis on the characterization of nonlinearities of superconductors.

Dr. Mateu was the recipient of the 2004 Prize for the best doctoral thesis in fundamental and basic technologies for information and communications and their applications presented by the Colegio Oficial de Ingenieros de Telecomunicación (COIT) and Asociación Española de Ingenieros de Telecomunicación (AEIT). He was also the recipient of a Fulbright Research Fellowship and a Ramón y Cajal Contract.

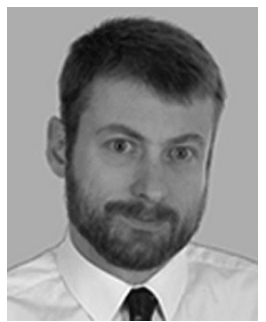

Juan M. O'Callaghan (SM'01) was born in Tortosa, Spain, in 1963. He received the Telecommunication Engineering degree from the Universitat Politècnica de Catalunya (UPC), Barcelona, Spain, in 1987 and the M.S. and Ph.D. degrees from the University of Wisconsin-Madison, in 2000 and 2003, respectively.

From July to December 1989, he was an Intern with the Systems Research Center, Honeywell. In 1993, he joined the faculty of UPC, where he has been teaching courses on radar, radio-navigation, and high-frequency devices and systems. Since May 2003, he has been a Full Professor with UPC . His research interests include microwave devices and systems, including high-frequency applications of superconductors. 